\title{
Correlações genéticas entre medidas de perímetro escrotal e características produtivas e reprodutivas de fêmeas da raça Nelore
}

\author{
Arione Augusti Boligon ${ }^{1}$, Paulo Roberto Nogara Rorato², Lucia Galvão de Albuquerque ${ }^{3}$ \\ 1 Mestranda em Genética e Melhoramento Animal, FCAVIUNESP - Jaboticabal/SP. Bolsista FAPESP.
2 Departamento de Zootecnia, UFSM - Santa Maria/RS. \\ ${ }^{3}$ Departamento de Zootecnia, FCAV/UNESP - Jaboticabal/SP.
}

RESUMO - Com objetivo de estimar parâmetros genéticos para perímetro escrotal medido aos 12 (PE12) e aos 18 meses de idade (PE18) de machos e peso a um ano de idade (P365), peso ao primeiro parto (PPP), idade ao primeiro parto (IPP), dias para o primeiro parto (DP) e duração da primeira gestação (DG) medidos em fêmeas, além de correlações entre perímetro escrotal e as demais características, foram analisadas medidas de 9.932 fêmeas e medidas de perímetro escrotal de 14.521 machos Nelore provenientes de 28 rebanhos das regiões Sul e Sudeste do País. Os componentes de (co)variância foram estimados utilizando-se o método da máxima verossimilhança restrita, sob modelo animal, em análises bi-características. As herdabilidades estimadas foram altas para PE12 (0,25 a 0,26), PE18 (0,35 a 0,37), P365 (0,35 e 0,38), PPP $(0,40$ e 0,41$)$ e DG $(0,19$ e 0,20$)$ e de menor magnitude para IPP $(0,14$ e 0,15$)$ e DP $(0,05$ e 0,06$)$. As correlações genéticas estimadas entre PE12 e P365, PPP, IPP, DP e DG foram de 0,$28 ; 0,30 ;-0,15 ;-0,04$ e 0,10 , respectivamente. As correlações genéticas entre PE18 e P365, PPP, IPP, DP e DG, no entanto, foram de 0,$29 ; 0,32 ;-0,21 ;-0,08$ e 0,14 , respectivamente. Esses resultados sugerem melhorias na eficiência reprodutiva de fêmeas, principalmente quando os rebanhos são selecionados para PE18.

Palavras-chave: fertilidade, herdabilidade, idade ao primeiro parto, parâmetros genéticos, peso

\section{Genetic correlations between male scrotal circumference and female productive and reproductive traits in Nellore cattle}

\begin{abstract}
Genetic parameters for scrotal circumference at 12 (SC12) and at 18 months of age (SC18) of males, and yearling weight (W365), weight at first calving (WFC), age at first calving (AFC), days to first calving (DC) and length of first gestation (GL) of females were estimated using records from 9,932 females and 14,521 males from 28 Nellore herds, located in the South and Southeast regions of Brazil. Covariance components were estimated by REML, fitting bivariate animal models. Heritability estimates were high for SC12 (0.25 to 0.26), SC18 (0.35 to 0.37), W365 (0.35 and 0.38), WFC (0.40 and 0.41$)$ and GL (0.19 and 0.20), but low for AFC (0.14 and 0.15) and DC (0.05 and 0.06). Genetic correlation estimates between SC12 and W365, WFC, AFC, DC and GL were $0.28,0.30,-0.15,-0.04$ and 0.10 , respectively. In the same order, the respective genetic correlation estimates between SC18 and female traits were $0.29,0.32,-0.21,-0.08$ and 0.14 . These results suggest that selection to improve female fertility efficiency based on SC18 would be more effective than by using SC12.
\end{abstract}

Key Words: age at first calving, fertility, genetic parameters, heritability, weight

\section{Introdução}

Entre as características importantes a serem consideradas nos sistemas de produção de carne bovina, destacam-se a eficiência reprodutiva e a taxa de crescimento. Segundo Silveira et al. (2004), a eficiência reprodutiva dos rebanhos é um dos fatores determinantes da eficiência total de produção e deve, portanto, ser considerada objetivo de seleção.

Vários autores relatam evidências de variação genética no desempenho reprodutivo de machos e fêmeas e relações favoráveis entre desempenho e características associadas ao desempenho ponderal, o que viabiliza a inclusão de características reprodutivas nos programas de seleção (Meyer et al., 1991; Mercadante et al., 2000; Forni \& Albuquerque, 2005).

Atualmente, a medida do perímetro escrotal é uma das características mais utilizadas como critério de seleção para melhorar a eficiência reprodutiva em gado de corte, principalmente por ser de fácil mensuração e por apresentar magnitude de herdabilidade média a alta (Silva et al., 2000; Pereira et al., 2002; Gianlorenço et al., 2003). Entretanto, por sua baixa correlação genética com algumas características medidas em fêmeas, como peso e data ao primeiro parto 
(Silva et al., 2000; Gressler et al., 2000, Pereira et al., 2002), a utilização do perímetro escrotal isoladamente talvez não seja a melhor alternativa para seleção de matrizes visando melhoria do desempenho reprodutivo.

Assim, em virtude da necessidade de mais informações para escolha das matrizes que serão mantidas e/ou introduzidas nos plantéis, visando melhorar a produção dos rebanhos de corte, vários autores têm estudado características que se expressam diretamente nas fêmeas e que sejam indicadoras de precocidade e fertilidade (Silva et al., 2000; Pereira et al., 2002; Dias et al., 2004; Azevêdo et al., 2006b). Entretanto, essas características são de difícil mensuração e interpretação, pois são decorrentes de interações entre bezerro, touro e vaca. Além disso, apresentam baixa herdabilidade (Mercadante et al., 2000; Dias et al., 2004).

A característica idade ao primeiro parto é, entre as características medidas nas fêmeas, a mais utilizada para avaliar a precocidade e a fertilidade, pois, segundo Azevêdo et al. (2006a), é de fácil mensuração e está relacionada à longevidade potencial da fêmea. Porém, Gressler et al. (2000) destacaram que essa medida pode não ser a mais adequada para a seleção e o descarte de matrizes bovinas e sugeriram o estudo de outras, como a data do parto e/ou dias para o parto.

Do mesmo modo, Pereira et al. (2000) sugeriram que a característica dias para o parto, comparação ao intervalo de partos, seria mais adequada para utilização em gado de corte, principalmente em decorrência do viés causado pela estação de monta fixa, geralmente de curta duração.

A duração da gestação, embora não seja propriamente uma característica de fertilidade, vem sendo considerada nas avaliações genéticas para melhorar o desempenho reprodutivo dos rebanhos, pois, de acordo com Pereira et al. (2002), está estreitamente relacionada ao período produtivo, influenciando o número de dias para o parto e até mesmo a idade ao primeiro parto.

Este estudo foi realizado com o objetivo de estimar parâmetros genéticos para característica de perímetro escrotal medido aos 12 e aos 18 meses de idade e características medidas em fêmeas (peso a um ano de idade, peso ao primeiro parto, idade ao primeiro parto, dias para o parto e duração da gestação), além de estimar a correlação genética entre as medidas de perímetro escrotal e as medidas produtivas e reprodutivas de fêmeas em rebanhos da raça Nelore.

\section{Material e Métodos}

As informações utilizadas referem-se a medidas de perímetro escrotal de 14.521 machos, filhos de 504 touros e 11.439 matrizes e características produtivas e reprodutivas de 9.932 fêmeas, filhas de 388 touros e de 6.130 matrizes, pertencentes a 28 rebanhos localizados nas regiões Sul e Sudeste do país. O período de observação das características foi de 1991 a 2000.

Para as análises, foram utilizados animais com pai e mãe conhecidos, produtos de inseminação artificial, nascidos de parto simples e criados em regime exclusivo de pastagem. $\mathrm{O}$ grupo de contemporâneos foi restringido para que o tamanho fosse composto de, no mínimo, cinco animais. A matriz de parentesco continha informações de 27.141 animais.

As características avaliadas foram: perímetro escrotal aos 12 (PE12) e aos 18 meses de idade (PE18) dos machos; e peso a um ano de idade (P365); peso ao primeiro parto (PPP); idade ao primeiro parto (IPP); dias para o primeiro parto (DP); duração da primeira gestação (DG) medidas nas fêmeas.

A IPP, em dias, foi obtida pela diferença entre a data do primeiro parto e a data do nascimento da fêmea. A característica de DP corresponde ao número de dias entre o primeiro dia da estação de monta e a data do parto subseqüente. A DG foi calculada pela diferença, em dias, da data de inseminação artificial em relação à data do parto.

As novilhas foram submetidas à reprodução aos 27 meses de idade aproximadamente. Os valores de PE foram obtidos por meio de medições realizadas nos animais aos 12 e aos 18 meses de idade. Foram consideradas duas estações de nascimento (dezembro a maio e junho a novembro) e duas estações de monta (março a maio e outubro a dezembro).

Foram realizadas análises de variância, pelo método dos quadrados mínimos, utilizando-se o procedimento GLM(SAS, 2001). A partir deste estudo inicial, cujo resultado indicou os efeitos ambientais que estavam influenciando significativamente $(\mathrm{P}<0,05)$ as características a serem avaliadas, foram definidos os grupos de contemporâneos e as covariáveis a serem incluídas nos modelos de avaliação genética.

Os componentes de (co)variância utilizados para estimar os parâmetros genéticos para todas as características avaliadas e as correlações genéticas entre as mensurações de perímetro escrotal aos 12 e aos 18 meses e as características produtivas e reprodutivas das fêmeas foram obtidos pelo método da máxima verossimilhança restrita livre de derivadas, empregando-se modelo animal, com a utilização do aplicativo MTDFREML (Boldman et al., 1995), em análises bi-características.

Os efeitos fixos considerados para cada característica são descritos a seguir:

PE12: grupo de contemporâneos (incluindo os animais nascidos na mesma fazenda, em mesmo ano e estação, e pertencentes ao mesmo grupo de manejo ao ano), além do efeito linear daidade do animal na mensuração, como covariável. 
PE18: grupo de contemporâneos (incluindo os animais nascidos na mesma fazenda, em mesmo ano e estação e pertencentes ao mesmo grupo de manejo ao sobreano), além do efeito linear e quadrático da idade do animal na mensuração, como covariável.

P365: grupo de contemporâneos (incluindo animais nascidos na mesma fazenda, em mesmo ano e estação, e pertencentes ao mesmo grupo de manejo ao ano).

PPP: grupo de contemporâneos (incluindo animais nascidos na mesma fazenda, no mesmo ano, pertencentes ao mesmo grupo de manejo ao sobreano, estação de monta e ano de parição) e sexo do bezerro.

IPP: grupo de contemporâneos (incluindo os animais nascidos na mesma fazenda e ano, pertencentes ao mesmo grupo de manejo ao sobreano e estação de monta).

DP e DG: grupo de contemporâneos (incluindo os animais nascidos na mesma fazenda e no mesmo ano, pertencentes ao mesmo grupo de manejo ao sobreano, estação de monta e ano de parição) e sexo do bezerro, como covariável. Considerou-se a idade da vaca no início da estação de monta (efeito linear e quadrático).

Para estimativa das correlações entre as medidas de perímetro escrotal e as características produtivas e reprodutivas observadas nas fêmeas, considerou-se o seguinte modelo bi-característica:

$$
\left[\begin{array}{l}
y_{1} \\
y_{2}
\end{array}\right]=\left[\begin{array}{cc}
X_{1} & 0 \\
0 & X_{2}
\end{array}\right]\left[\begin{array}{l}
b_{1} \\
b_{2}
\end{array}\right]+\left[\begin{array}{cc}
Z_{1} & 0 \\
0 & Z_{2}
\end{array}\right]\left[\begin{array}{l}
u_{1} \\
u_{2}
\end{array}\right]+\left[\begin{array}{l}
e_{1} \\
e_{2}
\end{array}\right]
$$

em que: $\mathrm{y}_{1}=$ vetor dos registros de produção da característica 1 (P365; PPP; IPP; DP; DG); $\mathrm{y}_{2}=$ vetor dos registros de produção para a característica 2 (PE12; PE 18); $\mathrm{b}_{1}=$ vetor dos efeitos fixos para característica $1 ; \mathrm{b}_{2}=$ vetor dos efeitos fixos para característica $2 ; \mathrm{u}_{1}=$ vetor dos efeitos aleatórios de valor genético para característica 1 ; $\mathrm{u}_{2}=$ vetor dos efeitos aleatórios de valor genético para característica $2 ; \mathrm{X}_{1} \mathrm{e} \mathrm{X}_{2}=$ matriz de incidência associando elementos de $b_{1}$ e $b_{2}$ a $_{1}$ e $y_{2} ; Z_{1} e Z_{2}=$ matriz de incidência

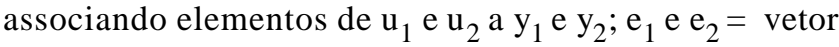
dos efeitos residuais

Para o modelo bi-característica, em geral, $\mathrm{E}\left[y_{i}\right]=\mathrm{X}_{\mathrm{i}} \mathrm{b}_{\mathrm{i}}$ para i $=1$ e 2 ; e a matriz de variância-covariância dos elementos aleatórios no modelo é determinada por:

$$
\operatorname{Var}\left[\begin{array}{c}
u_{1} \\
u_{2} \\
e_{1} \\
e_{2}
\end{array}\right]=\left[\begin{array}{cccc}
A \sigma_{a 1}^{2} & A \sigma_{a 1 a 2} & 0 & 0 \\
A \sigma_{a 1 a 2} & A \sigma_{a 2}^{2} & 0 & 0 \\
0 & 0 & I \sigma_{e 1}^{2} & 0 \\
0 & 0 & 0 & I \sigma_{e 2}^{2}
\end{array}\right]
$$

em que: $\mathrm{A}=$ matriz de parentesco; $\sigma_{a 1}^{2}, \sigma_{a 2}^{2}=$ variância genética aditiva para as características 1 e 2 , respectivamente; $\sigma_{a 1 a 2}=$ covariância genética aditiva entre as características 1 e $2 ; \sigma_{e 1}^{2}, \sigma_{e 2}^{2}=$ variância ambiental para as características 1 e 2 , respectivamente.

\section{Resultados e Discussão}

Os resultados da análise descritiva das características utilizadas neste estudo são apresentados na Tabela 1. O valor médio observado para PE12 foi semelhante ao descrito por Gianlorenço et al. (2003), que relataram média de 20,45 $\mathrm{cm}$ para a raça Canchim. Para PE18, a média obtida neste estudo se assemelha aos valores descritos na literatura, que variam de 25,55 a 27,75 cm para a raça Nelore (Ortiz Pena et al., 2001; Pereira et al., 2002; Silveira et al., 2004; Forni \& Albuquerque, 2005).

Os valores obtidos para características medidas em fêmeas se assemelham aos da literatura. Para P365 e PPP, Talhari et al. (2003) relataram médias de 205,2 e 429,0 kg, respectivamente. Do mesmo modo, a média obtida para P365 foi próxima ao valor descrito por Mercadante et al. (2000) e Bertazzo et al. (2004) para fêmeas da raça Nelore (187 e $225,46 \mathrm{~kg}$, respectivamente). A média da IPP se assemelha às relatadas por Mercadante et al. (2000), Pereira et al. (2002), Silveira et al. (2004) e Forni \& Albuquerque (2005), de $1.149,0 ; 1.053,2 ; 1.272,6$ e 1.064 dias, respectivamente, para raça Nelore. As médias obtidas para DG e DP foram similares às encontradas por Pereira et al. (2002) para raça Nelore (290,60 e 320,38 dias, respectivamente). Do mesmo modo, Forni \& Albuquerque et al. (2005) relataram média de 308 dias para DP para fêmeas da raça Nelore.

As estimativas dos componentes de (co)variâncias e dos parâmetros genéticos são descritas nas Tabelas 2 e 3 . Os componentes de variância estimados nas análises bi-característica envolvendo o perímetro escrotal aos $12 \mathrm{e}$ 18 meses de idade com características medidas em fêmeas foram semelhantes.

A herdabilidade média estimada para PE12 $(0,25)$ foi praticamente a mesma relatada por Meyer et al. (1991) e Gressler et al. (2000), cujos valores variaram de 0,24 a 0,26. Por outro lado, Eler et al. (1996), Silva et al. (2000) e Gianlorenço et al. (2003) obtiveram estimativas de herdabilidade superiores para PE12, com valores de 0,30 a 0,52 .

As herdabilidades estimadas para PE18 variaram de 0,35 a 0,37. Esses valores são semelhantes aos obtidos por Pereira et al. (2001), Dias et al. (2003) e Boligon et al. (2006), de 0,22 a 0,35 . Por outro lado, na maioria dos trabalhos citados na literatura, o PE18 apresentou herdabilidade 
Tabela 1 - Número de observações (N), média $(\bar{x})$, desvio-padrão (DP), coeficiente de variação (CV), valores mínimo (MIN) e máximo (MAX) e número de grupos contemporâneos (NGC) para as características avaliadas

Table 1 - Number of records $(N)$, average $(\bar{x})$, standard deviation (SD), coefficient of variation (CV), minimum and maximum values and number of contemporary groups (NCG) of each trait

\begin{tabular}{|c|c|c|c|c|c|c|c|}
\hline $\begin{array}{l}\text { Característica } \\
\text { Trait }\end{array}$ & $\mathrm{N}$ & $\bar{x}$ & $\begin{array}{l}\mathrm{DP} \\
S D\end{array}$ & $\begin{array}{l}\mathrm{CV}(\%) \\
\text { Minimum }\end{array}$ & $\begin{array}{c}\text { MIN } \\
\text { Maximum }\end{array}$ & $\begin{array}{c}\mathrm{MAX} \\
N C G\end{array}$ & NGC \\
\hline $\mathrm{PE} 12(\mathrm{~cm})(S C 12)$ & 14.521 & 20,13 & 3,49 & 17,34 & 13 & 25 & 321 \\
\hline PE18 (cm) (SC18) & 14.521 & 26,88 & 3,51 & 13,06 & 17 & 37 & 319 \\
\hline P365（kg) (W365) & 9.932 & 210,71 & 41,98 & 19,92 & 166,99 & 294,21 & 372 \\
\hline PPP (kg) (WFC) & 9.932 & 402,93 & 49,31 & 12,24 & 311,06 & 490,07 & 412 \\
\hline IPP (dias) (AFC, days) & 9.932 & $1.104,3$ & 111,9 & 10,13 & 723,45 & $1.738,7$ & 386 \\
\hline $\mathrm{DP}$ (dias) $(D C$, days $)$ & 9.932 & 315,21 & 18,91 & 5,99 & 281,32 & 379,90 & 412 \\
\hline DG (dias) $(G L$, days $)$ & 9.932 & 291,08 & 27,11 & 9,31 & 271,31 & 306,14 & 412 \\
\hline
\end{tabular}

PE12 e PE18 (perímetro escrotal medido aos 12 e 18 meses); P365 (peso aos 365 dias de idade, fêmeas); PPP (peso ao primeiro parto); IPP (idade ao primeiro parto); DP (dias para o primeiro parto) e DG (duração da primeira gestação).

SC12 and SC18 (scrotal circumference at 12 and at 18 months of age); W365 (female yearling weight); WFC (weight at first calving); AFC (age at first calving); DC (days to first calving) and $\mathrm{GL}$ (length of first gestation).

superior à encontrada neste estudo, de 0,39 a 0,52, para raça Nelore (Eler et al., 1996; Pereira et al., 2000; Ortiz Peña et al., 2001; Pereira et al., 2002; Forni \& Albuquerque, 2005). Segundo Pereira et al. (2001), baixas estimativas de herdabilidade para perímetro escrotal podem ser decorrentes de inadequado ajuste do perímetro escrotal pela idade do animal na medição.

Comparando as herdabilidades estimadas para PE12 e PE18, verifica-se a existência de maior variabilidade genética para PE18, indicando que esta idade é a mais adequada para seleção pelo perímetro escrotal nessa população. Resultado semelhante foi obtido por Gressler et al. (2000), porém com menor diferença entre as duas estimativas de herdabilidade.

As herdabilidades estimadas para P365 $(0,35$ e 0,38$)$ e PPP $(0,40$ e 0,41$)$ sugerem que essas características respondem à seleção de forma satisfatória. Resultados semelhantes são relatados na literatura e variam de 0,35 a 0,40 (Mercadante et al., 2000; Bertazzo et al., 2004) para P365 e de 0,33 a 0,42 (Silva et al., 2000; Talhari et al., 2003) para PPP.

As herdabilidades estimadas para a IPP foram de baixa magnitude $(0,14$ e 0,15$)$, o que pode ser explicado, em parte, pelo fato de que somente as fêmeas que pariram foram consideradas nas análises. Desse modo, mesmo utilizando um arquivo de genealogia completo, muitas vezes não é possível obter estimativa acurada da variabilidade genética na população. Esses resultados se assemelham à maioria dos descritos na literatura, que variam de 0,08 a 0,21 (Pereira et al., 2000; Silva et al., 2000; Pereira et al., 2001; Dias et al., 2003; Azevêdo et al., 2006b). Por outro lado, é inferior aos relatados por Mercadante et al. (2000), de 0,26 e 0,27 para IPP em análises bi-características com peso ajustado aos 240 e 365 dias de idade, respectivamente.
Os valores estimados para os coeficientes de herdabilidade para as características DP e DG estão descritos nas Tabelas 2 e 3. Herdabilidade similar à estimada neste estudo para DP foi relatada por Pereira et al. (2002), Mercadante et al. (2003) e Forni \& Albuquerque (2005), de 0,04 a 0,07. Entretanto, para a DG, a herdabilidade estimada condiz com a amplitude descrita na literatura, ou seja, 0,12 a 0,26 (Pereira et al., 2002; Azevêdo et al., 2006b). A herdabilidade estimada para DG permite classificar como positiva a resposta à seleção para essa característica.

As baixas herdabilidades estimadas para DP sugerem pequena variabilidade genética aditiva na expressão dessa característica, mais influenciada por condições ambientais. Segundo Forni \& Albuquerque (2005), baixa estimativa de herdabilidade para DP também pode ser reflexo da utilização de fêmeas que não emprenharam nas avaliações e/ou efeito de mudanças na magnitude das variâncias genéticas e ambientais durante a vida reprodutiva do animal.

Segundo Pereira et al. (2002), a utilização de características com herdabilidades inferiores a 0,14 em programas de melhoramento teria como conseqüência pequena resposta à seleção e exigiria que os reprodutores tivessem grande número de filhas avaliadas para que as DEPs apresentassem confiabilidade suficiente. Entretanto, é importante avaliar as características reprodutivas, mesmo que de baixa herdabilidade, quando se pretende selecionar matrizes jovens, pois esta seleção possibilita a obtenção mais rápida de retorno do capital investido, aumentando a vida reprodutiva e o número de progênies por vaca.

As correlações genéticas positivas entre mensurações de perímetro escrotal e características produtivas medidas em fêmeas indicam que a seleção para aumento do perímetro escrotal acarretará, por meio de seleção indireta, aumento do 
Tabela 2 - Estimativas dos componentes de (co)variância e dos parâmetros genéticos considerando o perímetro escrotal medido aos 12 meses de idade (característica 1) e características medidas em fêmeas (característica 2): peso a um ano de idade (P365), peso ao primeiro parto (PPP), idade ao primeiro parto (IPP), dias para o parto (DP) e duração da gestação (DG), em análises bi-características

Table 2 - Estimates of (co)variance components and genetic parameters for males scrotal circumference at 12 months of age (trait 1) and female traits (trait 2): yearling weight (W365), weight at first calving (WFC), age at first calving (AFC), days to calving (DC) and length of first gestation length (GL), from bivariate analyses

\begin{tabular}{|c|c|c|c|c|c|c|}
\hline \multirow[t]{2}{*}{$\begin{array}{l}\text { Parâmetro } \\
\text { Parameter }\end{array}$} & \multicolumn{6}{|c|}{$\begin{array}{l}\text { Perímetro escrotal aos } 12 \text { meses de idade (1) } \\
\text { Scrotal circumference at } 12 \text { months of age (1) }\end{array}$} \\
\hline & $\begin{aligned} \text { P365 (2) } \\
\text { W365 (2) }\end{aligned}$ & $\begin{array}{l}\text { PPP (2) } \\
W F C(2)\end{array}$ & $\begin{array}{l}\text { IPP (2) } \\
F C(2)\end{array}$ & $\begin{array}{c}\mathrm{DP}(2) \\
D C(2)\end{array}$ & $\begin{array}{l}\text { PIP (2) } \\
F C I(2)\end{array}$ & $\begin{array}{l}\text { DG (2) } \\
G L(2)\end{array}$ \\
\hline$\sigma_{a}^{2} 1$ & 2,05 & 2,06 & 2,06 & 2,09 & 2,03 & 2,06 \\
\hline$\sigma^{2}{ }_{a} 2$ & 301,09 & 528,02 & 693,06 & 96,31 & 51,09 & 9,03 \\
\hline$\sigma_{\mathrm{a}} 1,2$ & 6,36 & 9,11 & $-11,09$ & $-1,79$ & $-2,25$ & 0,46 \\
\hline$\sigma_{e}^{2} 1$ & 6,03 & 6,45 & 6,22 & 6,13 & 6,21 & 6,29 \\
\hline$\sigma_{\mathrm{e}}^{2} 2$ & 588,21 & 985,11 & $5.607,48$ & $1.829,89$ & 851,33 & 33,99 \\
\hline$\sigma_{p}^{2} 1$ & 8,08 & 8,51 & 8,28 & 8,22 & 8,24 & 8,35 \\
\hline$\sigma_{p}^{2} 2$ & 889,30 & $1.513,13$ & $6.300,54$ & $1.926,20$ & 902,42 & 43,02 \\
\hline$h^{2} 1$ & 0,25 & 0,24 & 0,25 & 0,25 & 0,25 & 0,25 \\
\hline$h^{2} 2$ & 0,34 & 0,35 & 0,11 & 0,05 & 0,06 & 0,21 \\
\hline$r_{a}$ & 0,26 & 0,28 & $-0,13$ & $-0,13$ & $-0,22$ & 0,11 \\
\hline
\end{tabular}

$\overline{\sigma^{2}}{ }_{a} 1=$ variância genética aditiva para característica $1 ; \sigma^{2}{ }_{a} 2=$ variância genética aditiva para características $2 ; \sigma_{\mathrm{a}} 1,2=$ covariância genética entre características 1 e $2 ; \sigma^{2}{ }_{e} 1=$ variância ambiental para característica $1 ; \sigma^{2}{ }_{e} 2=$ variância ambiental para características $2 ; \sigma^{2}{ }^{2} 1=$ variância fenotípica para característica $1 ; \sigma^{2}{ }_{2} 2=$ variância fenotípica para características $2 ; h^{2} 1=$ herdabilidade para característica $1 ; h^{2} 2=$ herdabilidade para características 2 ; $\mathrm{r}_{\mathrm{a}}=$ correlação genética entre característica 1 e 2 .

$\sigma^{2}{ }_{a} 1=$ aditive genetic variance for trait $1 ; \sigma^{2}{ }_{a}^{2}=$ aditive genetic variance for traits $2 ; \sigma_{a} 1,2=$ genetic covariance between traits 1 and $2 ; \sigma^{2}{ }_{e} 1=$ environmental variance for trait $1 ; \sigma^{2} e_{e}^{2}=$ environmental variance for traits $2 ; \sigma^{2}{ }_{p} 1=$ phenotypic variance for trait $1 ; \sigma^{2}{ }_{p}^{2}=$ phenotypic variance for trait $2 ; \sigma_{p} ; h^{2} 1=$ heritability for trait $1 ; h^{2} 2=$ heritability for trait $2 ; r_{a}=$ genetic correlation between traits 1 and 2 .

Tabela 3 - Estimativas dos componentes de (co)variância e dos parâmetros genéticos considerando o perímetro escrotal medido aos 18 meses de idade (característica 1) e características medidas em fêmeas (característica 2): peso a um ano de idade (P365), peso ao primeiro parto (PPP), idade ao primeiro parto (IPP), dias para o parto (DP), primeiro intervalo de partos (PIP), duração da gestação (DG), em análises bi-características

Table 3 - $\quad$ Estimates of (co)variance components and genetic parameters for males scrotal circumference at 18 months of age (trait 1) and female traits (trait 2): yearling weight (W365), weight at first calving (WFC), age at first calving (AFC), days to calving (DC) and length of first gestation length (GL), from bivariate analyses

\begin{tabular}{|c|c|c|c|c|c|c|}
\hline \multirow[t]{2}{*}{$\begin{array}{l}\text { Parâmetro } \\
\text { Parameter }\end{array}$} & \multicolumn{6}{|c|}{$\begin{array}{l}\text { Perímetro escrotal aos } 18 \text { meses de idade (1) } \\
\text { Scrotal circumference at } 18 \text { months of age (1) }\end{array}$} \\
\hline & $\begin{array}{c}\text { P365 (2) } \\
\text { W365 (2) }\end{array}$ & $\begin{array}{l}\text { PPP (2) } \\
W F C \quad(2)\end{array}$ & $\begin{array}{c}\text { IPP (2) } \\
F C(2)\end{array}$ & $\begin{array}{c}\mathrm{DP}(2) \\
D C(2)\end{array}$ & $\begin{array}{c}\text { PIP (2) } \\
F C I(2)\end{array}$ & $\begin{array}{c}\text { DG (2) } \\
G L(2)\end{array}$ \\
\hline$\sigma_{a}^{2} 1$ & 3,15 & 2,99 & 3,06 & 3,14 & 2,81 & 3,11 \\
\hline$\sigma_{a}^{2} 2$ & 321,14 & 498,10 & 702,15 & 102,31 & 41,54 & 10,74 \\
\hline$\sigma_{\mathrm{a}} 1,2$ & 9,01 & 10,91 & $-10,66$ & $-1,95$ & $-3,76$ & 1,64 \\
\hline$\sigma_{\mathrm{e}}^{2} 1$ & 6,23 & 6,01 & 5,98 & 5,99 & 8,82 & 6,16 \\
\hline$\sigma_{\mathrm{e}}^{2} 2$ & 571,19 & 952,04 & $4.902,21$ & $1.526,63$ & 298,91 & 31,95 \\
\hline$\sigma_{p}^{2} 1$ & 9,38 & 9,00 & 9,04 & 9,13 & 11,63 & 9,27 \\
\hline$\sigma_{p}^{2} 2$ & 892,33 & $1.450,14$ & $5.604,36$ & $1.628,94$ & 340,45 & 42,69 \\
\hline$h^{2} 1$ & 0,33 & 0,33 & 0,34 & 0,34 & 0,35 & 0,33 \\
\hline$h^{2} 2$ & 0,36 & 0,34 & 0,14 & 0,06 & 0,12 & 0,25 \\
\hline$r_{a}$ & 0,28 & 0,28 & $-0,23$ & $-0,11$ & $-0,35$ & 0,28 \\
\hline
\end{tabular}

$\sigma^{2}{ }_{a} 1=$ variância genética aditiva para característica $1 ; \sigma^{2}{ }_{a} 2=$ variância genética aditiva para características $2 ; \sigma_{\mathrm{a}} 1,2=$ covariância genética entre característicaS 1 e $2 ; \sigma^{2}{ }_{e} 1=$ variância ambiental para característica $1 ; \sigma^{2}{ }^{2} 2=$ variância ambiental para características $2 ; \sigma^{2}{ }^{2} 1=$ variância fenotípica para característica $1 ; \sigma^{2}{ }_{2} 2=$ variância fenotípica para características $2 ; h^{2} 1=$ herdabilidade para característica $1 ; h^{2} 2=$ herdabilidade para características 2 ; $r_{2}=$ correlação genética entre característica 1 e 2 .

$r_{2}=$ correlaçao genetica entre característica 1 e 2 .
$\sigma_{a}^{2}{ }_{1} 1=$ aditive genetic variance for trait $1 ; \sigma^{2}{ }_{a} 2=$ aditive genetic variance for traits $2 ; \sigma_{a} 1,2=$ genetic covariance between traits 1 and $2 ; \sigma_{e}^{2}{ }_{e} 1=$ environmental variance for trait $1 ; \sigma^{2}{ }_{e}^{2}=$ environmental variance for traits $2 ; \sigma^{2}{ }_{p} 1=$ phenotypic variance for trait $1 ; \sigma^{2}{ }_{p}^{2}=$ phenotypic variance for trait $2 ; \sigma_{p} ; h^{2} 1=$ heritability for trait $1 ; h^{2} 2=$ heritability for trait $2 ; r_{a}=$ genetic correlation between traits 1 and 2 . 
peso a um ano de idade e ao primeiro parto nas fêmeas. Além disso, estudando rebanhos da raça Canchim, Silva et al. (2000) relataram que a seleção para perímetro escrotal não interferiu no peso adulto das fêmeas, o que é altamente favorável.

As correlações genéticas da IPP com PE12 e PE18 foram negativas e de baixa a média magnitude (Tabelas 2 e 3 ). Resultado semelhante foi relatado por Pereira et al. (2002) para as idades de 14 e 26 meses com PE18 (-0,39 e -0,19, respectivamente), demonstrando que a seleção de reprodutores para maior perímetro escrotal, fornece, em resposta correlacionada, menor idade ao primeiro parto de sua progênie e que esta redução na IPP pode ser mais rápida quando se selecionam machos para PE18 em relação ao PE12.

A correlação genética entre DP e PE12 foi praticamente nula e indica que a seleção para maior perímetro escrotal, medido em idades jovens, não acarretará modificações consideráveis nos DP. Por outro lado, a correlação genética favorável obtida entre DP e PE18 indica que a seleção para maior perímetro escrotal medido ao sobreano, a longo prazo, pode causar redução no DP nos rebanhos avaliados, semelhante ao relatado por Pereira et al. (2002) e Forni \& Albuquerque (2005). Esses resultados permitem sugerir a inclusão de DP em programas de seleção visando melhorias genéticas para precocidade sexual e fertilidade.

Por outro lado, a correlação genética entre DG e PE18, superior a relatada por Pereira et al. (2002), sugere que parte dos genes responsáveis pelo maior desenvolvimento escrotal é também responsável pela DG. Assim, a seleção de reprodutores com maior PE18 promoveria gestações mais longas na progênie, o que não é desejável.

As correlações genéticas obtidas entre PE18 x DP $(-0,08)$ e PE18 x DG $(0,14)$ apresentaram resultados divergentes, uma vez que a maior parte da DP contém a DG. Nos rebanhos estudados, as fêmeas que apresentaram gestação mais longa foram, na maioria, as mesmas que emprenharam no início da estação de monta, o que pode explicar, em parte, correlação positiva entre PE18 e DG e praticamente nula entre PE18 e DP.

\section{Conclusões}

Os coeficientes de herdabilidade estimados para idade ao primeiro parto e dias para o parto indicam a possibilidade de obtenção de pequeno ganho genético com a inclusão destas características nos programas de seleção.

As correlações genéticas favoráveis entre o perímetro escrotal (mensuradas em diferentes idades) e características medidas em fêmeas, com exceção da DG, permitem a utilização do perímetro escrotal como critério de seleção visando melhorar a eficiência reprodutiva nas fêmeas.

Nas condições deste estudo, a seleção para maior perímetro escrotal aos 18 meses de idade pode resultar, a longo prazo, em menor idade ao primeiro parto, o que é desejável.

\section{Literatura Citada}

AZEVÊDO, D.M.M.R.; MARTINS FILHO, R.; BOZZI, R. et al. Parâmetros genéticos e fenotípicos do desempenho reprodutivo de fêmeas Chianina. Revista Brasileira de Zootecnia, v.35, n.3, p.982-987, 2006a (supl. 3)

AZEVÊDO, D.M.M.R.; MARTINS FILHO, R.; LÔBO, R.N.B. et al Desempenho reprodutivo de vacas Nelore no Norte e Nordeste do Brasil. Revista Brasileira de Zootecnia, v.35, n.3, p.988996, 2006b (supl. 3).

BERTAZZO, R.P.; FREITAS, R.T.F.; GONÇALVES, T.M. et al. Parâmetros genéticos de longevidade e produtividade de fêmeas da raça Nelore. Revista Brasileira de Zootecnia, v.33, n.5, p.1118-1127, 2004

BOLDMAN, K.G.; KRIESE, L.A.; Van VLECK, L.D. et al. A manual for use of MTDFREML: a set of programs to obtain estimates of variance and (co)variance (DRAFT). Lincoln: Department of Agriculture/ARS, 1995. 120p.

BOLIGON, A.A.; RORATO, P.R.N.; WEBER,T. et al. Herdabilidades para ganho de peso da desmama ao sobreano e perímetro escrotal ao sobreano e tendência genética e fenotípica para ganho de peso da desmama ao sobreano em bovinos Nelore-Angus. Revista Brasileira de Zootecnia, v.35, n.4, p.1323-1328, 2006.

DIAS, L.T.; EL FARO, L.; ALBUQUERQUE, L.G. Estimativas de herdabilidade para perímetro escrotal de animais da raça Nelore. Revista Brasileira de Zootecnia, v.32, n.6, p.1878-1882, 2003 (supl. 2)

DIAS, L.T.; EL FARO, L.; ALBUQUERQUE, L.G. Estimativas de herdabilidade para idade ao primeiro parto de novilhas da raça Nelore. Revista Brasileira de Zootecnia, v.33, n.1, p.97102,2004

ELER, J.P.; FERRAZ, J.B.S.; SILVA, P.R. Parâmetros genéticos para peso, avaliação visual e circunferência escrotal na raça Nelore, estimados por modelo animal. Arquivo Brasileiro de Medicina Veterinária e Zootecnia, v.48, n.2, p.203-213, 1996.

FORNI, S.; ALBUQUERQUE, L.G. Estimates of genetic correlations between days to calving and reproductive and weight traits in Nelore cattle. Journal of Animal Science, v.83, p.1511$1515,2005$.

GIANLORENÇO, V.K.; ALENCAR, M.M.; TORAL, F.L.B. et al Herdabilidades e correlações genéticas de características de machos e fêmeas, em um rebanho bovino da raça Canchim. Revista Brasileira de Zootecnia, v.32, n.6, p.1587-1593, 2003 (supl. 1)

GRESSLER, L.S.; BERGMANN, J.A.G.; PEREIRA, C.S. et al. Estudo das associações genéticas entre perímetro escrotal e características reprodutivas de fêmeas Nelore. Revista Brasileira de Zootecnia, v.29, n.2, p.427-437, 2000.

MERCADANTE, M.E.Z.; LÔBO, R.B.; OLIVEIRA, H.N. Estimativas de (co)variância entre características de reprodução e de crescimento em fêmeas de um rebanho Nelore. Revista Brasileira de Zootecnia, v.29, n.4, p.997-1004, 2000.

MERCADANTE, M.E.Z.; PACKER, I.U.; RAZOOK, A.G. et al Direct and correlated responses to selection for yearling weight on reprodutive performance of Nelore cows. Journal of Animal Science, v.81, p.376-384, 2003. 
MEYER, K.; HAMMOND, K.; MACKINNON, M.J. et al. Estimates of covariances between reproduction and growth in Australian beef cattle. Journal of Animal Science, v.69, n.8, p.3533$3543,1991$.

ORTIZ PEÑA, C.D.; QUEIROZ, S.A.; FRIES, L.A. Comparação entre critérios de seleção de precocidade sexual e a associação destes com características de crescimento em bovinos Nelore. Revista Brasileira de Zootecnia, v.30, n.1, p.39-100, 2001.

PEREIRA, E.; ELER, J.P.; FERRAZ, J.B.S. Correlação genética entre perímetro escrotal e algumas características reprodutivas na raça Nelore. Revista Brasileira de Zootecnia, v.29, n.6, p.1676-1683, 2000.

PEREIRA, E.; ELER, J.P.; COSTA, F.A.A. et al. Análise genética da idade ao primeiro parto e do perímetro escrotal em bovinos da raça Nelore. Arquivo Brasileiro de Medicina Veterinária e Zootecnia, v.53, n.1, p.116-121, 2001.

PEREIRA, E.; ELER, J.P.; FERRAZ, J.B.S. et al. Análise genética de características reprodutivas na raça Nelore. Pesquisa Agropecuária Brasileira, v.37, n.5, p.703-708, 2002.

STATISTICAL ANALYSIS SYSTEMS - SAS. User's guide: Version 6.11 ed. Cary: 2001.
SILVA, A.M.; ALENCAR, M.M.; FREITAS, A.R. et al. Herdabilidades e correlações genéticas para peso e perímetro escrotal de machos e características reprodutivas e de crescimento de fêmeas, na raça Canchim. Revista Brasileira de Zootecnia, v.29, n.6, p.2223-2230, 2000 (supl. 2).

SILVEIRA, J.C.; McMANUS, C.; MASCIOLI, A.S. et al. Fatores ambientais e parâmetros genéticos para características produtivas e reprodutivas em um rebanho no estado do Mato Grosso do Sul. Revista Brasileira de Zootecnia, v.33, n.6, p.1432-1444, 2004

TALHARI, F.M.; ALENCAR, M.M.; MASCIOLI, A.S. Correlações genéticas entre características produtivas de fêmeas em um rebanho da raça Canchim. Revista Brasileira de Zootecnia, v.32, n.4, p.880-886, 2003. 